\title{
Phosphatidylcholine Coatings Deliver Local Antimicrobials and Reduce Infection in a Murine Model: A Preliminary Study
}

\author{
Michael A. Harris BSBE, Karen E. Beenken PhD, Mark S. Smeltzer PhD, \\ Warren O. Haggard PhD, J. Amber Jennings PhD
}

Published online: 3 January 2017

(C) The Association of Bone and Joint Surgeons (B) 2016

\begin{abstract}
Background Phosphatidylcholine coatings have been shown to elute antibiotics for several days. A recently developed biofilm inhibitor, cis-2-decenoic acid (C2DA), has been shown to exhibit synergistic activity with several common antibiotics. This study aims to evaluate the effectiveness of C2DA and amikacin dual drug delivery from a phosphatidylcholine coating.

Questions/purposes (1) What are the in vitro elution profiles of amikacin and C2DA from phosphatidylcholinecoated coupons in incubated phosphate-buffered saline? (2)

Funded by the University of Memphis, Department of Biomedical Engineering (JAJ, WOH). Materials used in this study were supplied by Zimmer Biomet (Warsaw, IN, USA) during a previous collaboration.

All ICMJE Conflict of Interest Forms for authors and Clinical Orthopaedics and Related Research ${ }^{\circledR}$ editors and board members are on file with the publication and can be viewed on request.

Clinical Orthopaedics and Related Research ${ }^{\mathbb{R}}$ neither advocates nor endorses the use of any treatment, drug, or device. Readers are encouraged to always seek additional information, including FDAapproval status, of any drug or device prior to clinical use.

Each author certifies that his or her institution approved the animal protocol for this investigation and that all investigations were conducted in conformity with ethical principles of research. The in vivo animal model was performed at the University of Arkansas of Medical Sciences, Little Rock, AR, USA. All other work was performed at the University of Memphis, Memphis, TN, USA.
\end{abstract}

M. A. Harris ( $\square$ ), W. O. Haggard, J. A. Jennings

The University of Memphis, Department of Biomedical

Engineering, 330 Engineering Technology, 3796 Norriswood

Avenue, Memphis, TN 38152, USA

e-mail: Mhrris18@memphis.edu

K. E. Beenken, M. S. Smeltzer

Department of Microbiology \& Immunology and Department of Orthopaedics, The University of Arkansas for Medical Sciences, Little Rock, AR, USA
Does the presence of C2DA in eluate samples lower the amount of amikacin needed for bacterial inhibition in overnight bacterial turbidity assays? (3) Does addition of amikacin and C2DA result in decreased colony-forming units (CFUs) on wire implants and bone when compared with phosphatidylcholine coatings alone in a mouse model of periprosthetic joint infection?

Methods Effects of loading concentrations were assessed during 7-day in vitro elution studies for coatings containing all mixtures of $0 \%, 5 \%, 15 \%$, and $25 \%$ wt of amikacin and C2DA $(n=4)$ through quantitative high-performance liquid chromatography concentration determination and plotting concentration eluted over time. Antimicrobial activity was assessed by overnight turbidity testing of elution study samples against Staphylococcus aureus or Pseudomonas aeruginosa. In vivo efficacy was assessed using phosphatidylcholine-coated wire implants in a murine (mouse) model of infection $(n=3)$. Wire implants were coated with phosphatidylcholine containing no antimicrobials, amikacin alone, C2DA alone, or amikacin and C2DA and then inserted into the intramedullary femur of each mouse and inoculated with $S$ aureus. The number of viable bacterial colonies on the implant surface and in the surrounding bone was determined after 1 week with the goal of achieving complete bacterial clearance. Total viable CFU count and proportion of samples achieving complete clearance were compared between groups.

Results Elution samples showed a burst response of amikacin and C2DA for 1 to 2 days with C2DA release continuing at low levels through Day 4. All tested eluate samples inhibited $P$ aeruginosa. Samples from coatings containing $25 \%$ amikacin or $15 \%$ amikacin and any amount of C2DA were able to inhibit $S$ aureus formation, but all coatings with 5\% amikacin or $15 \%$ amikacin but no C2DA were not inhibitory. All in vivo treatment groups 
achieved complete bacterial clearance on the wire implant, and the C2DA alone and amikacin alone coatings cleared all CFUs in bone (pin: phosphatidylcholine only one of three; amikacin three of three, C2DA three of three, amikacin + C2DA three of three, $p=0.04$ [Fisher's exact test]; bone: coating only: zero of three; amikacin: three of three; C2DA; three of three; C2DA + amikacin: one of three; $\mathrm{p}=$ 0.03 [Fisher's exact test]).

Conclusions Phosphatidylcholine coatings elute antimicrobials in vitro under infinite sink conditions for up to 4 days in phosphate-buffered saline and were able to reduce bacterial colonies in a preliminary in vivo model. Turbidity testing with eluate samples containing varying amounts of C2DA and amikacin agrees with previous studies showing synergy between them.

Clinical Relevance Used as an adjunctive to systemic therapy, C2DA-loaded phosphatidylcholine coatings have potential value as a prophylactic infection prevention measure. Future studies may include different antibiotics, animal studies with larger sample sizes and more controls, and advanced coating delivery methods.

\section{Introduction}

Bacteria in biofilm-based implant infections are up to $\times 1000$ more resistant to antimicrobials than their planktonic counterparts, making systemic antibiotic therapy ineffective in the treatment of periprosthetic joint infection (PJI) [3]. Local antibiotic delivery methods have been investigated for several decades as a means of increasing antimicrobial concentration at the site of the wound while keeping systemic levels low, avoiding potential side effects. Antibioticloaded polymethylmethacrylate (PMMA) cement has been used for both prevention and treatment of PJI and has been shown to reduce the risk of infection in orthopaedic procedures $[9,15,29]$. The primary disadvantage of PMMA is that it is not biodegradable, so a second procedure is often needed to remove the device. Biodegradable delivery systems have been developed that offer similar drug elution profiles but completely degrade over a specified time interval, including collagen fleece, calcium sulfate, polymers such as lactic acid, and chitosan [5, 29]. The phospholipid phosphatidylcholine (PC) has been used in both purified form and as a PC-sesame oil gel to deliver antibiotics with favorable reductions in bacterial colony-forming units (CFUs) during in vivo PJI models $[13,19]$. PC has already been approved by the FDA as an additive to demineralized bone matrix to enhance bone ingrowth, suggesting that it would not induce adverse effects when used for drug delivery applications.

Naturally occurring biofilm dispersal agents have gained attention as a result of their ability to revert biofilm communities back into the planktonic state, eliminating the antimicrobial tolerance conferred by the biofilm [11, 16, 17, 24]. Among the recently investigated dispersal agents, cis-2-decenoic acid (C2DA) has shown potential as a result of its cross-species and cross-kingdom activity, effectiveness at low concentrations, good cytocompatibility, synergistic activity with many antibiotics, and its ability to inhibit biofilm formation when added to planktonic bacteria [4, 14, 16-18, 23, 25]. Despite promising results, there is currently a research gap between in vitro studies and development of effective local delivery devices for in vivo use. There is also a lack of in vivo studies examining the antibiofilm efficacy of C2DA at surgical wounds or confirming in vivo synergistic responses to antibiotics. The amphipathic structure of PC may make it an ideal carrier of both a hydrophobic fatty acid like C2DA and water-soluble antibiotics. PC has been previously used to deliver amikacin [13], which exhibits synergistic activity with C2DA in vitro [18] and has been shown to be cytocompatible with osteoblasts at concentrations up to 2000 $\mu \mathrm{g} / \mathrm{mL}[22]$.

Therefore, we asked: (1) What are the in vitro elution profiles of amikacin and C2DA from phosphatidylcholinecoated coupons in incubated phosphate-buffered saline? (2) Does the presence of C2DA lower the amount of amikacin needed for bacterial inhibition in overnight bacterial turbidity assays? (3) Does addition of amikacin and C2DA result in decreased CFUs on wire and bone when compared with phosphatidylcholine coatings alone in a mouse model of PJI?

\section{Materials and Methods}

Elution of antibiotics was first evaluated using in vitro elution studies. Antimicrobial activity was confirmed by turbidity testing using the eluate samples. Coating efficiency then was tested in vivo using a murine model of PJI.

\section{Fabrication}

C2DA and amikacin (MP Biomedical, Santa Ana, CA, USA) were incorporated into $90 \mathrm{~g}$ Phospholipon ${ }^{\circledR}$ (Lipoid, Ludwigshafen, Germany) by mixing $0 \mathrm{~g}, 50 \mathrm{mg}, 150 \mathrm{mg}$, or $250 \mathrm{mg}$ C2DA and $0 \mathrm{~g}, 50 \mathrm{mg}, 150 \mathrm{mg}$, or $250 \mathrm{mg}$ amikacin with enough PC to achieve a total mass of $1 \mathrm{~g}$ [12]. Individual coatings were mixed at room temperature through a chopping and kneading process until the drug payload was homogeneously distributed throughout the PC. Drug-loaded PC mixtures were made 24 hours before study use and stored at $-8{ }^{\circ} \mathrm{C}$. 
Elution and Activity

In vitro drug elution studies were performed to determine the effects of drug loading levels on release profile before in vivo experiments. Six-well plate CellCrown ${ }^{\text {TM }}$ inserts (Scaffdex, Tampere, Finland) were fitted with 41- $\mu \mathrm{M}$ nylon filters and inserted into six-well plates. Three-quarter inch titanium coupons were cut in house; polished by successive wet grinding with 400, 800, and 1000 grit sandpaper; sonicated in acetone for 30 minutes; and autoclaved for 30 minutes at $121^{\circ} \mathrm{C}$. Each coupon was coated by dragging the phosphatidylcholine mixture across the implant surface under gentle pressure to leave a thin coating $(n=4$ per group). Each coupon was inserted into a separate CellCrown $^{\mathrm{TM}}$ fitted well and each well received $3 \mathrm{~mL}$ phosphate-buffered saline (PBS). An adhesive cover was applied to each well plate and each plate was wrapped heavily with parafilm. The plates were placed in a $37{ }^{\circ} \mathrm{C}$ incubator on a Belly Dancer ${ }^{\mathrm{TM}}$ shaker (IBI Scientific, Peosta, IA, USA). Samples were taken every 24 hours with complete media refreshment for 7 days.

Amikacin and C2DA concentration in the eluate samples were measured using high-performance liquid chromatography (Thermo Scientific ${ }^{\mathrm{TM}}$ Dionex $^{\mathrm{TM}}$ UltiMate $^{\circledR}$ 3000, Waltham, MA, USA) with a C18 column (Thermo Scientific BDS Hypersil ${ }^{\mathrm{TM}} \mathrm{C} 18150 \times 4.6 \mathrm{~mm}$, particle size $5 \mu \mathrm{m})$. C2DA was measured at $209 \mathrm{~nm}$ using an isocratic buffer of $50.4 \%$ acetonitrile, $21.6 \%$ tetrahydrofuran, and $28.1 \%$ of $1 \%$ phosphoric acid [6]. Amikacin concentration was determined using a charged aerosol detector (Dionex Corona $^{\mathrm{TM}} \mathrm{Veo}^{\mathrm{TM}}$ Charged Aerosol Detector RS; Thermo Scientific) with an isocratic buffer of $98 \% 55 \mathrm{mM}$ trifluoracetic acid, $1 \%$ acetonitrile, and $1 \%$ methanol [28].

PC eluate activity was determined using a turbidity growth inhibition assay. Staphylococcus aureus (UAMS-1) and Pseudomonas aeruginosa (PA-ATCC 27317) were grown overnight in tryptic soy broth (TSB). UAMS-1 was diluted 1:50 and PA-ATCC 27317 was diluted 1:200. Representative samples from each elution group were selected for turbidity testing. In a 96-well plate, each well received $130 \mu \mathrm{L}$ TSB, $20 \mu \mathrm{L}$ of the appropriate eluate sample, and $50 \mu \mathrm{L}$ of bacterial inoculum (a 1:10 final dilution for each eluate sample). Each sample was tested against UAMS-1 and PA-ATCC 27317 in triplicate. The top row of each plate contained controls with six inoculated (negative controls) and six uninoculated (positive controls) wells receiving PBS instead of the eluate sample. The plates were incubated overnight at $37{ }^{\circ} \mathrm{C}$ and then turbidity was measured as absorbance at $540 \mathrm{~nm}$ (ELx800; Biotek, Winooski, VT, USA). The cutoff value for growth inhibition was set at $10 \%$ of the difference between positive and negative controls, plain TSB and inoculated TSB without antimicrobials, respectively.

\section{In Vivo Efficacy}

In vivo efficacy was tested using a pilot murine study at the University of Arkansas for Medical Sciences. PC was sterilized by gamma radiation, C2DA was filter-sterilized with a $0.20-\mu \mathrm{m}$ syringe tip filter, and powdered amikacin was sterilized by brief exposure to UV light. Implant wires were autoclave-sterilized at $121{ }^{\circ} \mathrm{C}$ before use. PC mixtures with no antimicrobials, $5 \%$ wt amikacin, $10 \%$ wt C2DA, or $5 \%$ wt amikacin, and $10 \%$ wt C2DA were made using an aseptic technique 24 hours before the surgery and stored at $-8{ }^{\circ} \mathrm{C}$.

Eight- to 12-week-old C57BL/6 mice weighing 16 to 18 $\mathrm{g}$ were anesthetized with isoflurane and Avertin (0.4-0.6 $\mathrm{mg} / \mathrm{g})$. A small incision was made over the left knee and a hole was bored into the left distal femur using 26-gauge and then 23-gauge syringe needles. A sterile 1-cm x 0.6$\mathrm{mm}$ diameter stainless steel Kirschner wire was coated with the appropriate PC mixture ( $n=3$ /group) and inserted into the femur. The wound was inoculated with approximately $10^{4}$ CFUs of UAMS- 1 and closed. The animals were humanely euthanized after 1 week, at which time the wire implant and appropriate femur were removed to determine the number of viable bacterial CFUs on/in each. The quantity of attached bacterial colonies on wire implants was determined by sonicating and vortexing in sterile PBS, plating the PBS on agarose, and counting the number of colonies after overnight incubation at $37{ }^{\circ} \mathrm{C}$. The surrounding bone was homogenized and suspended in sterile PBS. The number of viable bacterial colonies in the bone was then counted by plating the homogenized solution on agarose, incubating overnight, and counting the number of bacterial colonies, similar to the process used for the wire implants. Bacterial clearance for an implant or bone was defined as a viable bacterial CFU count of zero.

SigmaPlot (Systat Software, Inc, San Jose, CA, USA) was used for all statistical analysis testing. Kruskal-Wallis analysis of variance (ANOVA) on ranks was used to test for differences in bacterial counts for each group, including control, the implants, and the surrounding tissue. Dunn's multiple comparison test versus controls was used for post hoc analysis. Fisher's exact test was used to test for differences in the proportion of complete bacterial clearance between nonantimicrobial control coatings and combined results of antimicrobial coatings. A significance level of $5 \%$ was used for determining statistical significance between groups. 


\section{Results}

The PC-coated coupons eluted C2DA and amikacin in a burst pattern over the first 1 to 2 days that tapered off by Day 3 (Fig. 1A). Amikacin elution stopped after Day 3 in most groups, but there was an increase in elution at Days 6 and 7 in some groups (Fig. 1B). C2DA eluted at a faster rate in coatings containing $15 \%$ or $25 \%$ wt amikacin (Fig. 1B). It is worth noting that only $48 \%$ of the incorporated C2DA and $57 \%$ of incorporated amikacin were recovered from the eluate samples.

The presence of C2DA in the eluate lowered the amount of amikacin needed to inhibit UAMS-1 growth; an eluate with no C2DA and $156 \mu \mathrm{g} / \mathrm{mL}$ amikacin was unable to inhibit UAMS-1 growth, but a sample with $19 \mu \mathrm{g} / \mathrm{mL}$ amikacin and $88 \mu \mathrm{g} / \mathrm{mL} \mathrm{C2DA}$ was inhibitory (Table 1).

The eluates from coatings containing 5\% wt amikacin did not inhibit UAMS-1 regardless of C2DA, and eluates from coatings with $15 \%$ amikacin and no C2DA were also uninhibitory. However, all eluates from coatings containing either $25 \%$ amikacin or $15 \%$ amikacin and any concentration of C2DA were able to inhibit UAMS-1. All tested eluates were effective against PA-ATCC 27317.

No attached bacterial colonies were detected on the wire implants from antimicrobial-loaded PC groups, which is lower than colonies found on coatings only (Fig. 2). The proportion of implant clearance was different between
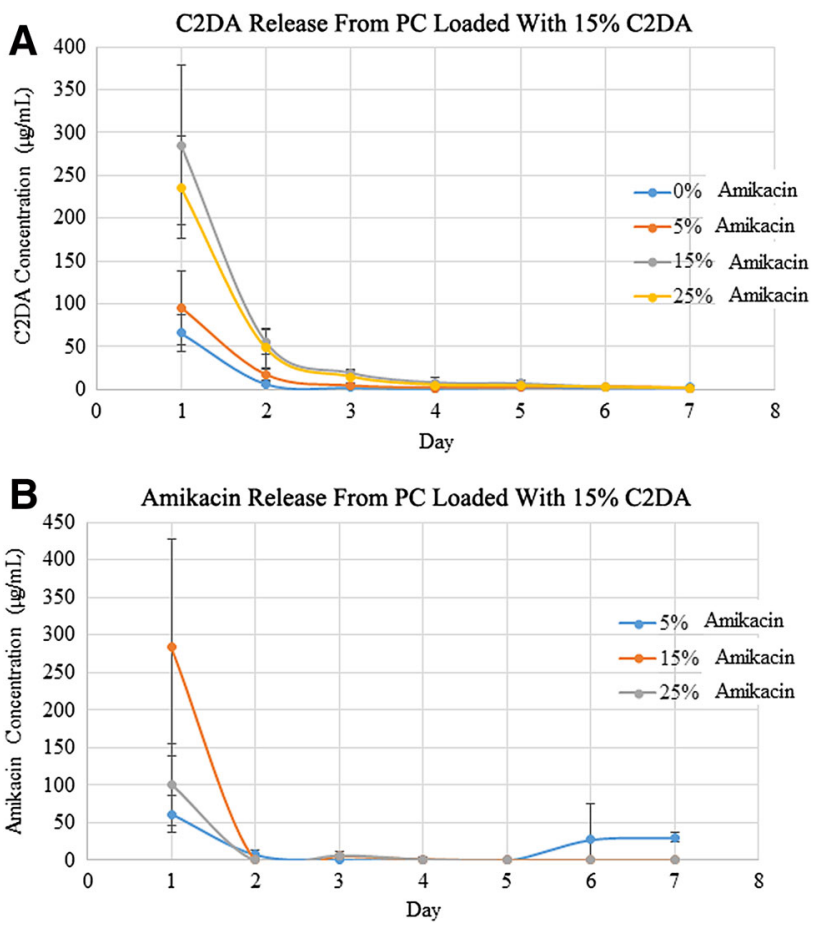

Fig. 1A-B Graphs show elution profiles for (A) C2DA and (B) amikacin from phosphatidylcholine coatings with $15 \%$ wt C2DA $($ mean $\pm \mathrm{SD})$. antimicrobial-loaded groups and unloaded PC (coating only: one of three; amikacin: three of three; C2DA: three of three; $\mathrm{C} 2 \mathrm{DA}+$ amikacin: three of three; $\mathrm{p}=0.045)$. The proportion of bone clearance was also higher between all antimicrobial-loaded groups and coating alone (coating only: zero of three; amikacin: three of three; C2DA; three of three; $\mathrm{C} 2 \mathrm{DA}+$ amikacin: one of three; $\mathrm{p}=0.03)$. There were differences in CFU counts on implants between the treatment groups and controls, although not statistically different at the $\mathrm{p}<0.05$ significance level using KruskalWallis ANOVA ( $\mathrm{p}=0.088)$. All nonloaded coatings had viable CFUs of bacteria in the surrounding bone (Fig. 2B). Kruskal-Wallis ANOVA detected a statistically significant difference in bacterial counts from the surrounding bone ( $p$ $=0.033$ ), and the amikacin alone and C2DA alone groups contained significantly less bacteria than the control group ( $\mathrm{p}=0.035)$. The C2DA and amikacin combination coating did not produce a significant reduction in bacterial count based off of ranked testing $(\mathrm{p}=0.620$; CFU count: coating only: $1.6^{*} 10^{6} \pm 2 * 10^{6}$; amikacin: $0 \pm 0$; C2DA: $0 \pm 0$; C2DA + amikacin: $8200 \pm 1 * 10^{4}$ CFUs).

\section{Discussion}

Local antibiotic delivery devices offer an effective method to achieve high local antibiotic concentrations at surgical sites for prophylactic treatment of biofilm-based PJI. Bacterial adhesion is often cited as the critical event in the establishment of biofilm on implant surfaces [2], making this process a potential target for future infection prevention strategies. The biofilm inhibitor C2DA has been previously shown to induce dispersion of mature biofilms, inhibit the formation of new biofilms from cultures of planktonic bacteria, inhibit bacterial growth at higher concentrations, and exert synergistic activity with common antibiotics. These properties suggest that C2DA may be able to inhibit biofilm formation on implants if delivered alongside antibiotics at the surgical site. There is a current gap in research between promising in vitro studies and the development of effective local drug delivery devices to administer them at a surgical wound bed. PC, a biocompatible and biodegradable phospholipid, may be used to incorporate various antimicrobials and applied to implant surfaces for local drug delivery. PC is advantageous for prophylactic implant protection because it rapidly degrades, can deliver a wide variety of drugs without the harsh loading or preparation steps seen in PMMA polymerization, and, as a coating, provides better implant coverage compared with collagen sponges that must rely on diffusion to deliver antimicrobials to the implant surface $[19,27]$. This work suggests that phosphatidylcholine coatings can effectively release amikacin and C2DA for 2 
Table 1. Eluate turbidity testing against Staphylococcus aureus and Pseudomonas aeruginosa

\begin{tabular}{|c|c|c|c|c|c|}
\hline Amikacin loading & C2DA loading & Measured amikacin $(\mu \mathrm{g} / \mathrm{mL})$ & Measured C2DA $(\mu \mathrm{g} / \mathrm{mL})$ & Inhibited UAMS-1 & Inhibited PA-ATCC 27317 \\
\hline Low & None & 156 & 0 & $\mathrm{~N}$ & $\mathrm{Y}$ \\
\hline Low & Low & 61 & 47 & $\mathrm{~N}$ & $\mathrm{Y}$ \\
\hline Low & Medium & 94 & 129 & $\mathrm{~N}$ & $\mathrm{Y}$ \\
\hline Low & High & 62 & 99 & $\mathrm{~N}$ & $\mathrm{Y}$ \\
\hline Medium & None & 65 & 0 & $\mathrm{~N}$ & $\mathrm{Y}$ \\
\hline Medium & Low & 19 & 88 & $\mathrm{Y}$ & Y \\
\hline Medium & Medium & 232 & 283 & $\mathrm{Y}$ & Y \\
\hline Medium & High & 254 & 150 & $\mathrm{Y}$ & $\mathrm{Y}$ \\
\hline High & None & 237 & 0 & $\mathrm{Y}$ & $\mathrm{Y}$ \\
\hline High & Low & 248 & 94 & $\mathrm{Y}$ & $\mathrm{Y}$ \\
\hline High & Medium & 158 & 291 & $\mathrm{Y}$ & $\mathrm{Y}$ \\
\hline High & High & 154 & 685 & $\mathrm{Y}$ & $\mathrm{Y}$ \\
\hline
\end{tabular}

Eluates were diluted 1:10 during the assay; $\mathrm{C} 2 \mathrm{DA}=$ cis-2-decenoic acid; $\mathrm{N}=$ no; $\mathrm{Y}=$ yes.

Fig. 2A-B Scatterplots show the number of viable bacterial CFUs recovered from (A) wire implants and (B) surrounding bone tissue (mean $\pm \mathrm{SD})$.
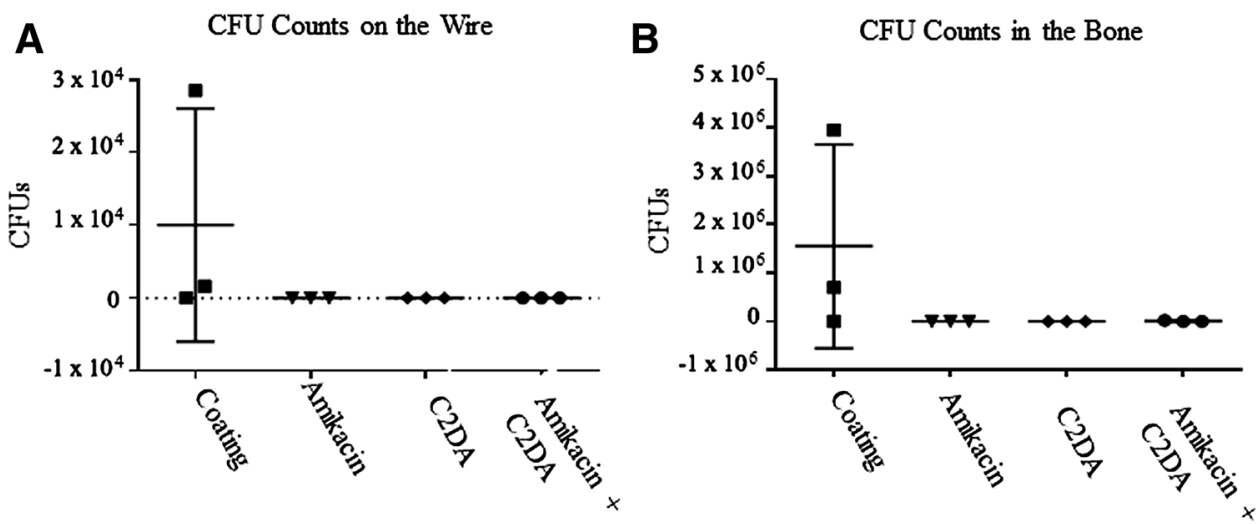

to 3 days from orthopaedic implants to prevent bacterial colonization. From a clinical perspective, these coatings have potential for prophylactic treatment of PJI.

Although the amikacin and C2DA-loaded PC coatings show promise in the animal model, there are some limitations with this study. The elution study method shows the drug elution pattern for our system but is not a fair representation of the various processes occurring in vivo, in which the degradation rate may be different. The animal model was only a pilot study with three animals per group and limited controls, making it impossible to draw firm conclusions about the effectiveness of the coating or local delivery of C2DA. The results of the in vitro turbidity assay and the in vivo bacterial counts are conflicting with combinations of C2DA and amikacin performing better in vitro, whereas the combination group was the only treatment group to test positive for bacteria in the surrounding bone in vivo. As a result of the preliminary nature of this study and limited sample size, it is inappropriate to make comparisons among the antibiotic, C2DA, and combination coatings; however, statistical analysis does suggest that drug-loaded coatings were significantly better than the PConly control. It is currently unknown how well these coatings will withstand aggressive implantation procedures or how coating will influence host-implant interactions, so these results may not translate into human studies. Although limited, the results do provide pilot data to support and plan further extended preclinical studies regarding the effectiveness of PC coatings and C2DA. It should also be noted that this coating is meant to rapidly degrade and will offer no protection from hematogenous seeding of bacteria from distant infections after it has degraded.

Elution study results suggest that PC coatings can elute amikacin and C2DA over several days with a rapid burst pattern over the first 2 days that quickly tapers off. The rapid burst of antibiotic will ensure high concentrations of antibiotic in the wound bed, and incorporation of C2DA may delay bacterial attachment long enough for antibiotics or the innate immune system to kill any invading bacteria. This burst release does, however, remove most of the drug 
payload quickly and shortens the overall effective elution period. The burst release also increases the chances of achieving cytotoxic levels of antimicrobials during the first day and therefore limits the amount of drug that may be incorporated. Interestingly, high-performance liquid chromatographic analysis shows only half of the incorporated antimicrobials were recovered in the eluate samples. This is likely related to bulk erosion of PC from the implant coating [10]. We believe that, during the initial elution period, large chunks of PC will erode from the coating and become dispersed in the PBS media as liposomes with encased C2DA and amikacin. These liposomes were removed when the media was changed daily, and the antimicrobials were not detected by the high-performance liquid chromatography because they were still encased in PC. This is advantageous because the liposomes will remain in the wound bed and continue to elute antimicrobials as the PC hydrolyzes and degrades, further extending the elution pattern beyond what was determined by highperformance liquid chromatography. During elution of amikacin, a second burst release was observed for the 5\% amikacin group at Days 6 and 7, which has been seen in previous antibiotic elution studies using PC [7]. It was previously suggested that this is related to PC hydrolysis; however, additional research is needed to understand why this phenomenon was not observed in groups with higher antibiotic loading concentrations.

C2DA has also shown synergistic effects with many common antibiotics and will lower the amount of drug needed to kill bacteria $[18,21]$, which is reflected in the results of our turbidity testing. Coatings loaded with only $15 \%$ amikacin were unable to inhibit UAMS-1 growth; however, eluates from coatings with $15 \%$ amikacin and C2DA were inhibitory. This supports previous findings showing combinatorial effects between amikacin and C2DA. Coatings with 5\% amikacin were unable to inhibit UAMS-1 growth regardless of the presence of C2DA, suggesting that this level of loading is too low for clinical use. All tested samples were able to inhibit PA-ATCC 27317, regardless of the presence of C2DA. $S$ aureus and $P$ aeruginosa are the most common Gram-positive and Gram-negative pathogens present in orthopaedic infections, respectively $[1,8,26]$, suggesting that local C2DA and amikacin release can provide broad-spectrum protection against a variety of potential pathogens.

The in vitro findings are in accordance with the findings of our pilot animal study, in which all treatment groups were able to prevent bacterial attachment to the wire implant during the 1-week study. Although previous research shows that $500 \mu \mathrm{g} / \mathrm{mL}$ C2DA is needed to inhibit bacterial growth [14, 23], the C2DA-only coating was able to completely inhibit bacteria on the wire implant and in the surrounding bone. In vitro elution data suggest that the peak C2DA concentration should be approximately $100 \mu \mathrm{g} / \mathrm{mL}$ or $20 \%$ of the minimum inhibitory concentration. One possible explanation for the complete clearance of bacteria on the wire implant and in the bone tissue is that the presence of C2DA delayed bacterial attachment long enough for the murine innate immune system to respond. This finding does call into question the use of antibiotics, because the fatty acid was able to achieve complete bacterial clearance on the pin and in the surrounding bone. Turbidity testing with the eluate samples still suggests that there is a combinatorial or synergistic effect between amikacin and C2DA, and the limited sample size of the in vivo groups limits the validity of any observations between treatment groups. However, this finding was not expected and does warrant an additional in vivo study with increased sample sizes to provide more statistically relevant data. The presence of bacteria in the surrounding bone of a limited number of animals suggests that the distance of antibiotic elution may be limited to the tissue adjacent to the implant. Although the number of CFUs recovered from bone tissue in the combination group was lower than that of the controls, bacteria could rebound and cause infection. Nevertheless, the antimicrobial-loaded coatings were able to increase the proportion of bacterial clearance when compared with the control. Because this coating would likely be an adjunct preventive measure to systemic prophylactic antibiotics [20], this risk of infection in surrounding tissue will be minimized.

In conclusion, phosphatidylcholine coatings can be used as local drug delivery devices for prevention of bacterial colonization on implant surfaces. The high early antimicrobial elution, as seen with in vitro elution studies, kills any contaminating bacteria during the initial wound healing time period. Local delivery of C2DA may prevent bacterial attachment and increase antibiotic effectiveness, preventing colonization and formation of biofilm. Our pilot in vivo murine study supports these findings and warrants testing with more antibiotics, animal studies with larger sample sizes and additional controls, and improved methods of clinical application of coatings to implants.

Acknowledgments We thank Karen Troxel for guidance in fabrication methods and study design input.

\section{References}

1. Arciola C, Alvi F, An Y, Campoccia D, Montanaro L. Implant infection and infection resistant materials: a mini review. Int $J$ Artif Organs. 2005;28:1119-1125.

2. Arciola CR, Alvi FI, An YH, Campoccia D, Montanaro L. Implant infection and infection resistant materials: a mini review. Int J Artif Organs. 2005;28:1119-1125. 
3. Costerton J, Montanaro L, Arciola C. Biofilm in implant infections: its production and regulation. Int $J$ Artif Organs. 2005;28:1062-1068.

4. Davies DG, Marques CN. A fatty acid messenger is responsible for inducing dispersion in microbial biofilms. $J$ Bacteriol. 2009;191:1393-1403.

5. El-Husseiny M, Patel S, MacFarlane R, Haddad F. Biodegradable antibiotic delivery systems. Bone Joint J. 2011;93:151-157.

6. Genç M, Aslan A. Determination of trans-10-hydroxy-2-decenoic acid content in pure royal jelly and royal jelly products by column liquid chromatography. J Chromatogr A. 1999;839:265-268.

7. Grit M, Underberg WJ, Crommelin DJ. Hydrolysis of saturated soybean phosphatidylcholine in aqueous liposome dispersions. $J$ Pharm Sci. 1993;82:362-366.

8. Haenle M, Skripitz C, Mittelmeier W, Skripitz R. Economic impact of infected total knee arthroplasty. ScientificWorldJournal. 2012;2012:196515.

9. Hake ME, Young H, Hak DJ, Stahel PF, Hammerberg EM, Mauffrey C. Local antibiotic therapy strategies in orthopaedic trauma: practical tips and tricks and review of the literature. Injury. 2015;46:1447-1456.

10. Heller J, Baker R. Theory and practice of controlled drug delivery from bioerodible polymers. In: Baker R, ed. Controlled Release of Bioactive Materials. New York, NY, USA: Academic Press; 1980:1-18.

11. Jabra-Rizk M, Meiller T, James C, Shirtliff M. Effect of farnesol on Staphylococcus aureus biofilm formation and antimicrobial susceptibility. Antimicrob Agents Chemother. 2006;50:14631469.

12. Jennings JA, Beenken KE, Skinner RA, Meeker DG, Smeltzer MS, Haggard WO, Troxel KS. Antibiotic-loaded phosphatidylcholine inhibits staphylococcal bone infection. World J Orthop. 2016;7:467.

13. Jennings JA, Carpenter DP, Troxel KS, Beenken KE, Smeltzer MS, Courtney HS, Haggard WO. Novel antibiotic-loaded pointof-care implant coating inhibits biofilm. Clin Orthop Relat Res. 2015;473:2270-2282.

14. Jennings JA, Courtney HS, Haggard WO. Cis-2-decenoic acid inhibits $S$ aureus growth and biofilm in vitro: a pilot study. Clin Orthop Relat Res. 2012;470:2663-2670.

15. Jiranek WA, Hanssen AD, Greenwald AS. Antibiotic-loaded bone cement for infection prophylaxis in total joint replacement. J Bone Joint Surg Am. 2006;88:2487-2500.

16. Marques CN, Davies DG, Sauer K. Control of biofilms with the fatty acid signaling molecule cis-2-decenoic acid. Pharmaceuticals. 2015;8:816-835.
17. Marques CN, Morozov A, Planzos P, Zelaya HM. The fatty acid signaling molecule cis-2-decenoic acid increases metabolic activity and reverts persister cells to an antimicrobial-susceptible state. Appl Environ Microbiol. 2014;80:6976-6991.

18. Masters E, Harris M, Jenninges JA. Cis-2-Decenoic interacts with bacterial cell membranes to potentiate additive and synergistic responses against biofilm. J Bacteriol Mycol. 2016;3:1-8.

19. Penn-Barwell JG, Murray CK, Wenke JC. Local antibiotic delivery by a bioabsorbable gel is superior to PMMA bead depot in reducing infection in an open fracture model. $J$ Orthop Trauma. 2014;28:370-375.

20. Prokuski L. Prophylactic antibiotics in orthopaedic surgery. J Am Acad Orthop Surg. 2008;16:283-293.

21. Rahmani-Badi A, Sepehr S, Mohammadi P, Soudi MR, BabaieNaiej H, Fallahi H. A combination of cis-2-decenoic acid and antibiotics eradicates pre-established catheter-associated biofilms. J Med Microbiol. 2014;63:1509-1516.

22. Rathbone CR, Cross JD, Brown KV, Murray CK, Wenke JC. Effect of various concentrations of antibiotics on osteogenic cell viability and activity. J Orthop Res. 2011;29:1070-1074.

23. Rawson M, Haggard W, Jennings JA. Osteocompatibility of biofilm inhibitors. Open Orthop J. 2014;8:442-449.

24. Sanchez CJ, Akers KS, Romano DR, Woodbury RL, Hardy SK, Murray CK, Wenke JC. D-amino acids enhance the activity of antimicrobials against biofilms of clinical wound isolates of Staphylococcus aureus and Pseudomonas aeruginosa. Antimicrob Agents Chemother. 2014;58:4353-4361.

25. Sepehr S, Rahmani-Badi A, Babaie-Naiej H, Soudi MR. Unsaturated fatty acid, cis-2-decenoic acid, in combination with disinfectants or antibiotics removes pre-established biofilms formed by food-related bacteria. PLOS ONE. 2014;9:e101677.

26. Stefánsdóttir A, Johansson D, Knutson K, Lidgren L, Robertsson O. Microbiology of the infected knee arthroplasty: report from the Swedish Knee Arthroplasty Register on 426 surgically revised cases. Scand J Infect Dis. 2009;41:831-840.

27. Stinner DJ, Hsu JR, Wenke JC. Negative pressure wound therapy reduces the effectiveness of traditional local antibiotic depot in a large complex musculoskeletal wound animal model. J Orthop Trauma. 2012;26:512-518.

28. Stypulkowska K, Blazewicz A, Fijalek Z, Sarna K. Determination of gentamicin sulphate composition and related substances in pharmaceutical preparations by LC with charged aerosol detection. Chromatographia. 2010;72:1225-1229.

29. Zalavras CG, Patzakis MJ, Holtom P. Local antibiotic therapy in the treatment of open fractures and osteomyelitis. Clin Orthop Relat Res. 2004;427:86-93. 